\title{
AN EXPLICIT FORMULA FOR THE EULER ZIGZAG NUMBER IN TERMS OF STIRLING NUMBERS OF THE SECOND KIND
}

\author{
SUMIT KUMAR JHA
}

\begin{abstract}
In this note, we derive an explicit formula for the Euler zigzag number in terms of Stirling numbers of the second kind.
\end{abstract}

Definition 1. The $n$th Euler zigzag number, denoted by $A_{n}$, is the number of alternating permutations of the set $\{1,2, \cdots, n\}$.

Definition 2. The Stirling numbers of the second kind, denoted by $S(n, m)$, is the number of ways of partitioning a set of $n$ elements into $m$ nonempty sets.

We prove the following

Theorem 1. We have

$$
A_{r}=-\frac{4^{r}}{a_{r}} \sum_{k=1}^{r} \frac{(-1)^{k} S(r, k)}{k+1}\left(\frac{3}{4}\right)^{(k)},
$$

where

$$
a_{r}=\left\{\begin{array}{ll}
(-1)^{\frac{r-1}{2}}\left(1+2^{-r}\right) & \text { if } r \text { is odd } \\
(-1)^{\frac{r}{2}} & \text { if } r \text { is even }
\end{array},\right.
$$

and $(x)^{(n)}=(x)(x+1) \cdots(x+n-1)$ denotes the rising factorial.

Proof. In the article [1], the author proved the following

$$
B_{r+1}=-\frac{r+1}{4\left(1+2^{-(r+1)}\left(1-2^{-r}\right)\right)}\left(\sum_{k=1}^{r}(-1)^{k} \cdot \frac{S(r, k)}{k+1} \cdot\left(\frac{3}{4}\right)^{(k)}+4^{-r} E_{r}\right),
$$

where $B_{r}$ and $E_{r}$ denote the Bernoulli numbers, and the Euler numbers, respectively.

2010 Mathematics Subject Classification. 11B68.

Key words and phrases. Euler zigzag numbers, Stirling numbers of the second kind, Bernoulli numbers, Euler numbers. 
If we let $r$ to be odd in equation (2), then considering odd indexed Euler numbers are zero, we have

$$
B_{r+1}=-\frac{r+1}{4\left(1+2^{-(r+1)}\left(1-2^{-r}\right)\right)} \sum_{k=1}^{r}(-1)^{k} \cdot \frac{S(r, k)}{k+1} \cdot\left(\frac{3}{4}\right)^{(k)} .
$$

We know that, from [2], we have

$$
A_{r}=(-1)^{\frac{r-1}{2}} \frac{2^{r+1}\left(2^{r+1}-1\right) B_{r+1}}{r+1},
$$

for all odd $r$. As a result, we can conclude that

$$
\begin{array}{r}
\frac{4(-1)^{\frac{r-1}{2}} A_{r}}{2^{r+1}\left(2^{r+1}-1\right)}\left(1+2^{-(r+1)}\left(1-2^{-r}\right)\right)=\frac{(-1)^{\frac{r-1}{2}} A_{r}}{4^{r}}\left(1+2^{-r}\right) \\
=-\sum_{k=1}^{r}(-1)^{k} \cdot \frac{S(r, k)}{k+1} \cdot\left(\frac{3}{4}\right)^{(k)} .
\end{array}
$$

which proves our result when $r$ is odd.

Now, if we let $r$ be an even in equation (2), then considering the fact that odd indexed Bernoulli numbers are zero (except 1), we have

$$
E_{r}=-4^{r} \sum_{k=1}^{r}(-1)^{k} \cdot \frac{S(r, k)}{k+1} \cdot\left(\frac{3}{4}\right)^{(k)}
$$

We know that, from [2], we have

$$
A_{r}=(-1)^{\frac{r}{2}} E_{r}
$$

for all even $r$. This proves our result when $r$ is even.

\section{REFERENCES}

1. Jha, Sumit Kumar. A new explicit formula for Bernoulli numbers involving the Euler number. Mosc. J. Comb. Number Theory 8 (2019), no. $4,385-387$.

2. Stanley, Richard P. A survey of alternating permutations. Contemp. Math 531 (2010): 165-196.

International Institute of Information Technology, Hyderabad, India

E-mail address: kumarjha.sumit@research.iiit.ac.in 